\title{
SEEING DECONVOLUTION OF GLOBULAR CLUSTERS IN M31
}

\author{
O. BENDINELLI \\ Dipartimento di Astronomia, Università di Bologna, Via Zamboni 33, 40126 Bologna, Italy \\ G. Parmeggiani \\ Osservatorio Astronomico di Bologna, Via Zamboni 33, 40126 Bologna, Italy \\ F. ZavatTi \\ Dipartimento di Astronomia, Università di Bologna, Via Zamboni 33, 40126 Bologna, Italy
}

S. DJORGOVSKI ${ }^{\text {), b) }}$

Division of Physics, Mathematics, and Astronomy, California Institute of Technology, Pasadena, California 91125 Received 1 September 1989; revised 17 November 1989

\begin{abstract}
Seeing deconvolution of surface-brightness profiles using the regularized multi-Gaussian method is potentially a powerful method for extraction of morphological information from digital images. Here we illustrate this method by applying it to CCD images of six globular clusters in M31. We demonstrate that it is possible to make some judgments about the dynamical structure of M31 globulars, using goodseeing, deconvolved CCD images obtained from the ground; for example, whether they have post-corecollapse morphology or not. The effective resolution limit of the method is about $0.1-0.3$ arcsec, for the CCD images obtained in FWHM $\simeq 1$ arcsec seeing, and sampling of 0.3 arcsec/pixel. We also demonstrate the robustness of the method, i.e., its insensivity to the exact choices of the functional representation of the point-spread function, details of the deconvolution technique, etc. The situation is equivalent in terms of angular resolution and sampling to observing globular clusters in the Virgo Cluster (e.g., around M87) with the Hubble Space Telescope, and the methods demonstrated here may be applied to HST data in the future. Dynamical structure of globular clusters, e.g., propensity for the post-corecollapse morphology as a function of position in the host galaxy, reflects the global dynamical evolution of a globular cluster system, as was already demonstrated for our galaxy. Similar studies of large samples of globulars in M31 and other Local Group galaxies from the ground, and out to Virgo with the $H S T$, can provide valuable insights about the mass distribution in galaxies, and the formation and evolution of their globular cluster systems. This pilot study is a step in this direction.
\end{abstract}

\section{INTRODUCTION AND MOTIVATION}

Optimal extraction of morphological information from seeing-smeared astronomical images is a longstanding problem. Whereas it is generally recognized that some recovery of the high spatial frequency information is possible, such attempts are seldom made because of the perceived numerical difficulties. Such timidity is unnecessary: If there are suitable reference sources in the field, i.e., stars, which are unresolved and thus represent the images of the point-spread function (PSF), seeing deconvolution is possible to a limit determined by the signal-to-noise ratio $(\mathrm{S} / \mathrm{N})$ and the sampling. A wide range of methods is known that can be used to reconstruct the full two-dimensional image, e.g., Wiener filtering, maximum entropy, etc. (Brault and White 1971; Frieden 1972; Bracewell 1979; Gull and Daniel 1978; Wells 1980; Bryan and Skilling 1980; Narayan and Nityananda 1986; Weir 1987), and in a high $\mathrm{S} / \mathrm{N}$ setting, they can result in angular resolution improvement typically up to a factor of 2 . The limits are usually imposed by the numerical instabilities, coupled with the finite $\mathrm{S} / \mathrm{N}$ of the data.

Alternatively, for some scientific applications, it may be desirable to pose a more restricted problem, and then apply more powerful restoration techniques. For example, if one is interested in the radial shape of an azimuthally (circularly

\footnotetext{
a) Visiting Astronomer, Kitt Peak National Observatory, NOAO, operated by AURA, Inc., under contract with the National Science Foundation.

b) Alfred P. Sloan Foundation fellow.
}

or elliptically) averaged surface-brightness profile, the problem of seeing deconvolution is effectively reduced to one dimension, and in principle a higher gain in angular resolution is possible. A class of regularized deconvolution and deprojection methods has been developed in previous papers in order to address these kinds of problems (Bendinelli et al. 1982, 1984a, b, 1985, 1986, 1987a, b, 1988, 1989; Bendinelli 1989; and references therein; cf. Sec. III).

The structure of cores of globular clusters contains valuable information about the dynamical structure and evolution of these systems. The problem of the core collapse and postcollapse evolution has been one of the principle issues in the dynamics of stellar systems for the past few years; see, e.g., the review by Elson, Hut, and Inagaki (1987), or the proceedings edited by Goodman and Hut (1985), Grindlay and Philip (1988), or Merritt (1989). The primary observational evidence for core collapse is existence of a central power law cusp in the surface-brightness profile, with the slope $\sim-1$ (Hénon 1961), a morphology quite distinct from the flat-core King (1966) models (Djorgovski and King 1984, 1986; Lugger et al. 1987; Djorgovski 1988; etc.). Approximately $20 \%$ of all globular clusters in our galaxy show this characteristic morphology (Djorgovski and King 1986). Also, in our galaxy, it has been possible to analyze the dependence of the cluster core morphology on such global variables as the cluster position with respect to the distance to Galactic center Galactic plane, cluster luminosity, metallicity, etc. A number of interesting correlations were found, which support the view that tidal shocks from disk and bulge 
passages can substantially accelerate or even dominate the cluster evolution (Chernoff and Djorgovski 1989). The census of globular cluster core morphologies can thus serve as a probe of the global dynamics and structure of a galaxy and its globular cluster system.

Outside of our galaxy, surveys of core morphology for the Magellanic Clouds globulars have been conducted by Mateo (1987) and Meylan and Djorgovski (1987; and in preparation). Some post-core-collapse clusters were found, but the indications are that the frequency of such cores is smaller in the Clouds than in the galaxy, and that the few concentrated globulars there are close to the central bar of the LMC, in agreement with the tidal shocks picture advocated by Chernoff and collaborators (Chernoff, Kochanek, and Shapiro 1986; Chernoff and Shapiro 1987; Chernoff and Djorgovski 1989). It would certainly be very interesting to carry out such an investigation for the globular cluster system of M31, but the combination of distance and seeing effects makes that a difficult task, and some seeing compensation scheme is necessary.

In this paper we explore the possibility of studying the morphology of M31 globular clusters, using seeing-deconvolved CCD images obtained from the ground. Our purpose is twofold: We wish to illustrate the application of our deconvolution techniques to an interesting astrophysical problem, and find their practical limitations, and also to initiate a morphological study of the M31 globulars. In future publications, we will continue our study of the M31 globular clusters system, and explore further similar seeing deconvolution analysis of the cores of nearby galaxies.

\section{THE DATA AND THE PSF FITS}

The images used in this study were obtained using the TI No. $2800 \times 800$ prime-focus CCD camera on the Kitt Peak 4 $\mathrm{m}$ telescope, in the $V$ band, on the night of UT 1985 December 16. The typical seeing FWHM was about 0.9-1.2 arcsec for the duration of these observations, and the sampling was $0.298 \mathrm{arcsec} / \mathrm{pixel}$. Six M31 globulars were observed: Mayall II, Mayall IV, Hubble III, G213, G263, and G280
(Sargent et al. 1977; Fusi Pecci 1988; and references therein). A total of ten CCD frames were obtained with integration times in the range 20-100 s. The data were processed using standard techniques.

Surface-brightness profiles were extracted using circular azimuthal averaging as described by Djorgovski (1988), and also using elliptical isophote fits, as described, e.g., by Kent (1983), Lauer (1985a), or Djorgovski (1985). Some of the longer exposures had cluster centers close to the saturation; these were edited carefully, and only the linear, well-exposed parts of the profiles were used and combined with those from the shorter exposures, where the centers were exposed correctly. Since the angular extent of M31 globulars is small with respect to the frame size, sky subtraction was not a serious problem. Surface-brightness profiles of unsaturated stars in the same images were also extracted, and used for the PSFs. The data on clusters are presented graphically in Sec. V. Table I lists the observed surface-brightness profiles, normalized with the central observed surface brightness.

In general, the PSF in a digital image is dominated by the atmospheric and dome "seeing," i.e., smearing due to the atmospheric turbulence (Fried 1966; Young 1974; Woolf 1982; Coulman 1985; and references therein), and also includes components due to the optical telescope properties, imperfections of the primary mirror surface, and various other mechanical defects. These components are now reasonably well understood, but their complete mathematical description is rather complex; instead, some simple functional representation that fits the data (i.e., the observed PSF intensity profiles) is desirable for practical applications. Among the popular analytical approximations of long-exposure PSFs is the modified power law function used by Moffat (1969):

$$
S(r)=S_{0}\left[1+(r / \alpha)^{2}\right]^{-\beta} .
$$

Another popular representation is a sum of Gaussians (e.g., Brown 1973):

$$
S(r)=\sum_{i=1}^{n} A_{i} \exp \left(-r^{2} / 2 \sigma_{i}^{2}\right),
$$

\begin{tabular}{|c|c|c|c|c|c|c|c|c|c|c|c|}
\hline \multicolumn{2}{|c|}{ Mayall II } & \multicolumn{2}{|c|}{ Mayall IV } & \multicolumn{2}{|c|}{ Hubble III } & \multicolumn{2}{|r|}{ G213 } & \multicolumn{2}{|r|}{$\mathrm{G} 263$} & \multicolumn{2}{|r|}{$\mathrm{G} 280$} \\
\hline$r(")$ & $\log \left(I / I_{0}\right)$ & $r(")$ & $\log \left(I / I_{0}\right)$ & $r(")$ & $\log \left(I / I_{0}\right)$ & $r(")$ & $\log \left(I / I_{0}\right)$ & $r(")$ & $\log \left(I / I_{0}\right)$ & $r\left({ }^{\prime \prime}\right)$ & $\log \left(I / I_{0}\right)$ \\
\hline $\begin{array}{r}0.40 \\
0.75 \\
1.05 \\
1.34 \\
1.64 \\
1.93 \\
2.23 \\
2.53 \\
2.82 \\
3.12 \\
3.71 \\
4.60 \\
5.49 \\
6.38 \\
7.27 \\
8.17 \\
9.06 \\
9.95 \\
10.84 \\
11.73 \\
12.62 \\
13.51 \\
14.40\end{array}$ & $\begin{array}{l}-0.088 \\
-0.306 \\
-0.528 \\
-0.770 \\
-0.984 \\
-1.169 \\
-1.331 \\
-1.478 \\
-1.610 \\
-1.726 \\
-1.923 \\
-2.167 \\
-2.364 \\
-2.534 \\
-2.673 \\
-2.830 \\
-2.958 \\
-3.103 \\
-3.191 \\
-3.327 \\
-3.499 \\
-3.622 \\
-3.737\end{array}$ & $\begin{array}{r}0.25 \\
0.43 \\
0.55 \\
0.70 \\
0.89 \\
1.14 \\
1.45 \\
1.85 \\
2.36 \\
3.01 \\
3.83 \\
4.88 \\
6.22 \\
7.93 \\
10.10\end{array}$ & $\begin{array}{l}-0.065 \\
-0.153 \\
-0.178 \\
-0.301 \\
-0.349 \\
-0.517 \\
-0.722 \\
-0.969 \\
-1.241 \\
-1.498 \\
-1.815 \\
-2.143 \\
-2.399 \\
-2.721 \\
-3.027\end{array}$ & $\begin{array}{l}0.25 \\
0.43 \\
0.55 \\
0.70 \\
0.89 \\
1.14 \\
1.45 \\
1.85 \\
2.36 \\
3.01 \\
3.83 \\
4.88 \\
6.22 \\
7.93\end{array}$ & $\begin{array}{l}-0.012 \\
-0.092 \\
-0.141 \\
-0.243 \\
-0.426 \\
-0.559 \\
-0.800 \\
-1.074 \\
-1.380 \\
-1.716 \\
-1.086 \\
-2.447 \\
-2.862 \\
-3.291\end{array}$ & $\begin{array}{l}0.25 \\
0.43 \\
0.55 \\
0.70 \\
0.89 \\
1.14 \\
1.45 \\
1.85 \\
2.36 \\
3.01 \\
3.83 \\
4.88 \\
6.22 \\
7.93\end{array}$ & $\begin{array}{l}-0.082 \\
-0.132 \\
-0.256 \\
-0.325 \\
-0.568 \\
-0.750 \\
-1.020 \\
-1.312 \\
-1.602 \\
-1.972 \\
-2.420 \\
-2.843 \\
-3.218 \\
-3.425\end{array}$ & $\begin{array}{l}0.26 \\
0.45 \\
0.58 \\
0.75 \\
0.97 \\
1.26 \\
1.64 \\
2.13 \\
2.76 \\
3.59 \\
4.66 \\
6.05 \\
7.85 \\
10.18\end{array}$ & $\begin{array}{l}-0.104 \\
-0.145 \\
-0.321 \\
-0.310 \\
-0.532 \\
-0.754 \\
-1.047 \\
-1.352 \\
-1.673 \\
-1.996 \\
-2.340 \\
-2.841 \\
-3.298 \\
-3.590\end{array}$ & $\begin{array}{l}0.26 \\
0.44 \\
0.57 \\
0.74 \\
0.96 \\
1.24 \\
1.61 \\
2.08 \\
2.69 \\
3.49 \\
4.51 \\
5.84 \\
7.55\end{array}$ & $\begin{array}{l}-0.142 \\
-0.200 \\
-0.363 \\
-0.473 \\
-0.691 \\
-0.948 \\
-1.227 \\
-1.541 \\
-1.893 \\
-2.255 \\
-2.700 \\
-3.176 \\
-3.603\end{array}$ \\
\hline
\end{tabular}

TABLE I. Observed surface-brightness profiles. 
where $n$ is a small number, e.g., 3. The fraction of the total light contributed by the $i$ th component, $L_{i}$, is proportional to $A_{i} \sigma_{i}^{2}$. These functions are fitted to the observed star profiles extracted from the CCD images, or the observed profile constructed by King (1971), using the Newton-Gauss regularized (NGR) method. Both analytical approximations (Moffat and multi-Gaussian) give statistically equally good fits (Bendinelli et al. 1987a,1988,1989). Multi-Gaussian and Moffat function fits were obtained for the observed PSF profiles in each frame. The observed profiles and the multiGaussian fits are illustrated in Fig. 1. The fit parameters are listed in Table II.

\section{DECONVOLUTION TECHNIQUE}

It must be pointed out that the sum of Gaussians does not represent directly a mixture of physically distinct Gaussian
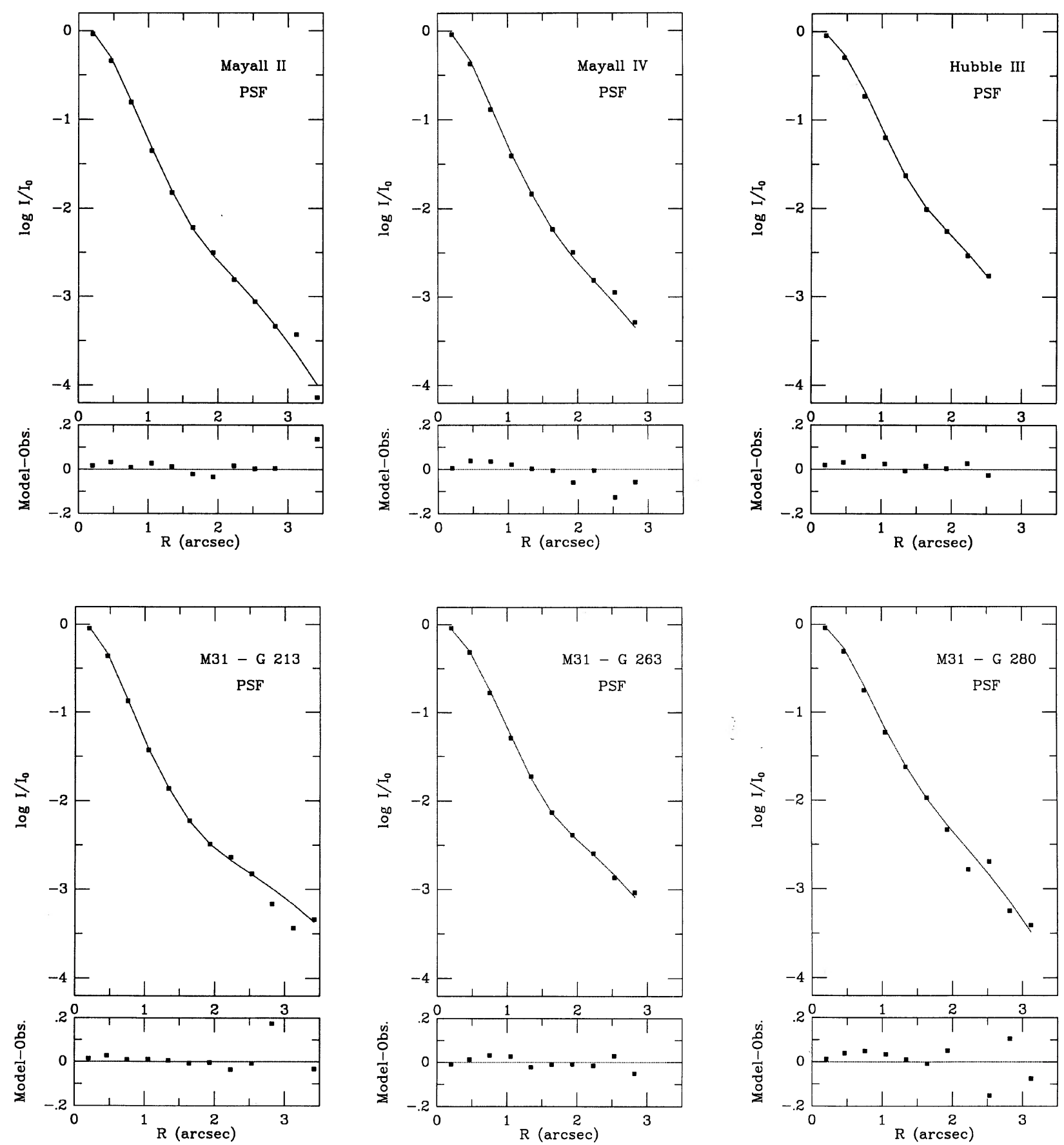

FIG. 1. Point-spread functions for the clusters. Dots represent the observed surface-brightness profiles, determined from bright, unsaturated stars in the CCD frames. Lines represent the multi-Gaussian fits to the data. Smaller panels show the residuals from the fits (note an expanded scale). 
TABLE II. Parameters of the fitted PSFs.

\begin{tabular}{cccccc}
\hline \hline \multirow{2}{*}{ Cluster } & $\begin{array}{c}\text { FWHM } \\
\text { (arcsec) }\end{array}$ & \multicolumn{2}{c}{$\begin{array}{c}\text { Three-Gaussian fit } \\
L_{i}, \sigma_{i}, i=1,2,3\end{array}$} & \multicolumn{2}{c}{$\begin{array}{c}\text { Moffat fit } \\
\alpha, \beta\end{array}$} \\
\hline Mayall II & 0.84 & 0.467 & 0.316 & 0.771 & 3.008 \\
& & 0.448 & 0.511 & & \\
Mayall IV & 0.83 & 0.575 & 0.323 & 0.595 & 2.443 \\
& & 0.343 & 0.549 & & \\
Hubble III & 0.94 & 0.306 & 0.335 & 0.860 & 3.026 \\
& & 0.539 & 0.482 & & \\
& & 0.156 & 1.054 & & \\
M31-G213 & 0.82 & 0.609 & 0.326 & 0.558 & 2.303 \\
& & 0.305 & 0.566 & & \\
& & 0.086 & 1.460 & & \\
M31-G263 & 0.90 & 0.364 & 0.326 & 0.665 & 2.516 \\
& & 0.524 & 0.495 & & \\
& & 0.113 & 1.168 & & \\
M31-G280 & 0.93 & 0.532 & 0.358 & 0.807 & 2.887 \\
& & 0.335 & 0.566 & & \\
& & 0.133 & 1.067 & & \\
\hline \hline
\end{tabular}

components, but rather it is a numerical approximation, viz., a truncation of an absolutely and rapidly convergent series. As shown by Bendinelli (1989), any smooth, monotonic distribution can be approximated by such a series of Gaussians (somewhat analogous to the representation of data as a Fourier series). Not only the PSF, but also the observed surface-brightness profiles of circularly symmetric astronomical objects (e.g., galactic cores or remote globular clusters) can be represented in this way. This multi-Gaussian approximation is the starting point that can simplify the discretization and solution of some numerically unstable inversion problems, formulated as integral equations of the first kind. Seeing deconvolution belongs to this class of inversion problems.

Following this idea, seeing deconvolutions of globular clusters in M31 have been done by a new numerical technique, regularized multi-Gaussian (RMG) method, which is described in more detail by Bendinelli (1989). The method utilizes the multi-Gaussian expansion of the PSF, and the assumption that the true light distribution $D(r)$, which is to be restored from the seeing-degraded data $C(r)$, can also be expanded in the same way, as a sum of Gaussians:

$$
D(r)=\sum_{j=1}^{m} B_{j} \exp \left(-r^{2} / 2 s_{j}^{2}\right),
$$

where the number of components, $m$, depends on the data in hand, and may be of the order of 10. By definition, the observed profile $C(r)$ is a two-dimensional convolution of the "true" profile $D(r)$, and the PSF $S(r)$, which is assumed to be represented by Eq. (2). As shown by Bendinelli (1989), the convolution integral equation has a simple analytical solution expressed by a double summation of Gaussian terms (modulo the renormalization factor):

$$
\begin{aligned}
C(r)= & \sum_{i=1}^{n} \sum_{j=1}^{m} \frac{A_{i} B_{j}}{\sigma_{i}^{2}+s_{j}^{2}} \exp \left[-r^{2} / 2\left(\sigma_{i}^{2}+s_{j}^{2}\right)\right] \\
& + \text { the noise. }
\end{aligned}
$$

This simplification is due to the beneficial properties of Gaussians, i.e., their separability. Deconvolution is thus reduced to a nonlinear estimation problem for the amplitudes $\boldsymbol{B}_{j}$ and the corresponding dispersions $s_{j}^{2}$, whose solution can be obtained numerically by the NGR method (Bendinelli et al. 1987a). As shown by Bendinelli (1989), this numerical problem can be simplified further, without loss of accuracy, if an additional constraint is introduced (a scaling relation for the dispersions $s_{j}^{2}$ of Gaussian terms in the deconvolved profile). The numerical stability of the solutions is assured by the regularization in the Tikhonov sense (Bendinelli et al. 1987a,1988,1989; Groetsch 1984).

The numerical accuracy of the solutions can be checked by computing the profile residuals in the sense (observed) - (deconvolved, and then convolved back by the observed PSF). Despite its simplicity, the RMG method produces residuals that are generally smaller than those obtained in deconvolutions using the unrestricted, regularized leastsquares (RLS) method. The latter is a deconvolution technique for circularly or elliptically symmetric light distributions (e.g., Bendinelli et al. 1986,1988), in which regularized least-squares solutions are obtained for large linear systems of equations. Typical rms values for the residuals are in the range 0.01-0.04 mag $\operatorname{arcsec}^{-2}$ for the RMG method, and $0.05-0.1$ mag $\operatorname{arcsec}^{-2}$ for the RLS method.

This apparent paradox that the residuals improve when using a less accurate mathematical description of the convolution integral, i.e., the truncated Gaussian series representation of the RMG versus the unrestricted profile shape in the RLS method, can be understood as follows: Recall that we are dealing with a highly unstable numerical inversion problem. In such cases, numerical instability grows rapidly with the dimension of the solution vector, and the same occurs for the bias introduced to stabilize the solution itself. Therefore, a solution represented by a small vector of parameters (as in the RMG method) produces residuals smaller than those given by a large numerical system of point-bypoint deconvolution with the RLS method. This illustrates a seldom appreciated point: In many inversion problems, seeing deconvolution included, the total errors can often be dominated by the numerical noise, rather than the noise present in the data. Therein lies the power of regularized methods like RLS or RMG. Also, it is worth mentioning that the RMG deconvolution is not critically dependent on the data sampling, in contrast to the RLS method and many other numerical deconvolution techniques.

\section{FEASIBILITY TESTS AND THE LIMITS OF THE METHOD}

A simple and basic feasibility test is to take a known answer, relevant for the problem at hand, degrade it to the observed seeing and $\mathrm{S} / \mathrm{N}$, and try to recover it using the same deconvolution technique as applied to the actual data. To do this, we "move" some well-observed Galactic globulars and suitably chosen artificial King model clusters to the distance to M31,* convolve the profiles with the observed PSF, resample the images with the same pixels as the actual data on the M31 globulars, and deconvolve using the same technique.

As suitable examples of Galactic clusters with postcollapse cores, we use M15 and NGC 6624. Their observed surface-brightness profiles, taken from Lugger et al. (1987), are well fitted as a composite of a modified Hubble profile, $I(r)=I(0)\left[1+\left(r / r_{\mathrm{c}}\right)^{2}\right]^{\gamma}$, with $\gamma \simeq-1$ (some authors er-

* We assume the distance to be $0.651 \mathrm{Mpc}$ (de Vaucouleurs 1978), giving the scale 1 acrsec $=3.16 \mathrm{pc}$, or $1 \mathrm{pc}=0.317$ arcsec. 
roneously call this a "King model," which it is not), and a central power law cusp. We use the composite fits by Lugger et al. (1987): in the case of M15, cusp with slope -0.64 grafted on a Hubble profile with core radius $r_{\mathrm{c}}=15.5$ arcsec, and in the case of NGC 6624, cusp with slope -0.77 grafted on a Hubble profile with $r_{\mathrm{c}}=14.2$ arcsec. We assumed distances of $9.7 \mathrm{kpc}$ to M15, and $8.0 \mathrm{kpc}$ to NGC 6624 (Webbink 1985), and rescaled the fits to the observed profiles to the assumed distance to M31. We also generated two pure King (1966) model clusters, with core radius $r_{\mathrm{c}}=0.05$ arcsec and concentration parameter $c=2.25$ (hereafter denoted as KM1), and with $r_{\mathrm{c}}=0.3$ arcsec and $c=1.5$ (KM2). These structural parameters correspond to a reasonable range observed for the Galactic globulars. All of these comparison cluster profiles were resampled with 0.298 arcsec pixels, convolved with the average PSF from the data on M31 globulars, and degraded with the artificial Poissonian noise so as to match or exceed the typical $\mathrm{S} / \mathrm{N}$ of our CCD data.

Seeing deconvolution of these degraded profiles was performed using the RMG method and the average PSF. The results (true profile, degraded profile, restored profile, and the residuals) for all four test cases are shown in Fig. 2. We see that the deconvolutions recover the morphological information to well below an arcsecond level. The two central cusps are recovered, and the restored profiles differ from the originals by about 0.08 in the power law slopes. The deviations of the restored profiles from the originals reach up to about 0.1 or 0.2 in log intensity, for M15 and NGC 6624, respectively, but are typically half as large. The two King models are restored with flat cores. The better-resolved one (KM2) has typical deviations of 0.04 in log intensity, and restored core radius of 0.28 arcsec. The more concentrated one (KM1) has deviations comparable to those for M15, and restored core radius of 0.14 arcsec.

This test demonstrates the feasibility of morphological measurements of globular clusters at the distance of M31, with the type of data described below. It also gives some indication of the limits of the technique and the data. We can probably distinguish the clusters with collapsed cores from King-model clusters, but the deduced power law slopes should not be trusted to better than about 0.1. Any deviations on the level of $\sim 0.1$ in log intensity over a decade in radius are within the expected errors. As for the measurements of core radii, they will generally be overestimated, and should be regarded as upper limits. If a restored core radius is comparable to the seeing HWHM or larger, it is recovered well. If it is several times smaller than the pixel size, it may be overestimated by a factor of 3 , or perhaps more. These numbers should guide the interpretation of results described below. They are also comparable to, or slightly better than, the practical limits to seeing deconvolutions obtained in other studies (cf. the references listed in Sec. I).

\section{SEEING DECONVOLUTIONS OF M31 GLOBULARS, AND THE ROBUSTNESS TESTS}

We obtained seeing-deconvolved profiles of the six M31 globulars, using the RMG method. The observed and the restored profiles for each cluster are shown in Fig. 3, along with the observed PSF data points and multi-Gaussian fits. For comparison, we also plot on the same $\log -\log$ scale a set of single-mass, isotropic, nonrotating King (1966) models.
Deconvolved profiles are listed in Table III, using the same surface-brightness normalization as in Table I.

Table IV gives some of the relevant parameters of the deconvolutions: observed and deconvolved HWHM in arcseconds, standard deviation of the first Gaussian component of the deconvolved profile (indicative of the limiting resolution), difference between observed and deconvolved central surface brightness, and the estimate of the numerical noise, expressed as the rms of the residuals (observed) - (deconvolved, and then convolved back by the observed PSF).

In addition to the tests described in Sec. IV, we can check how the results (i.e., the shape of the deconvolved profiles) depend on the functional form chosen for the PSF representation, and on the deconvolution method. For these internal tests, we use the data on Mayall II, the best observed and most luminous cluster in our sample.

We first test the dependence on the deconvolution method, and the representation of the PSF, viz., a single Gaussian $(\sigma=0.418$ arcsec), three-Gaussian (as used above), and Moffat modified power law, with the parameters listed in Table II. We use our RMG deconvolution as the fiducial one, and compare it with the RLS deconvolutions using the three PSFs. As before, the data are the circularly averaged surface-brightness profiles. The results are shown in Fig. 4, along with the residuals of RLS deconvolutions from the profile deconvolved with the RMG method. We see that maximum deviations reach up to 0.2 in $\log$ intensity, but are typically on the 0.05 level, or smaller. The three RLS deconvolutions are in good accord, never deviating more than about 0.05 in $\log$ intensity.

Next, we fix the form of the PSF as used above, viz., the multi-Gaussian with three components, and the parameters listed in Table II. We then perform deconvolutions using the RLS method with circular and with elliptical symmetry, by fixing the ellipticity to the mean observed value of 0.20 , and compare the results with our fiducial RMG deconvolution profile. The results are shown in Fig. 5. Given the different sampling between the circular and the elliptical cases, the profiles are in good agreement, with deviations similar to those obtained in the previous tests.

Again, we conclude that the limits of the deconvolution method with the data used here correspond to typical deviations of up to 0.1 in log intensity over one or two decades in radius. The maximal variations occur near 1 arcsec, which is the scale of the seeing disk.

\section{DISCUSSION}

Comparison of the deconvolved profiles shown in Fig. 3 with the King models, and the test cases shown in Fig. 2 suggest that none of the six clusters in this study have a collapsed core on the scale of the cusps seen in M15 and NGC 6624 in our galaxy. Smaller cusps are possible, and it should be noted that M15 and NGC 6624 are among the most dramatic cases of the post-core-collapse morphology known for Galactic globulars.

The deconvolved core radii (HWHM) are all in the range $\sim 0.5$ arcsec, or less. According to our tests in Sec. IV, this means that we probably have not resolved any of them, and that their true cores are probably at least a factor of 2 smaller, corresponding to $r_{\mathrm{c}}<1 \mathrm{pc}$. The most extreme case is Mayall II, where the true HWHM is probably not larger than 0.1 arcsec, corresponding to $r_{\mathrm{c}}<0.3 \mathrm{pc}$, and implying a high concentration, $c>2$. This cluster may be an M31 ana$\log$ of 47 Tuc in our galaxy. 

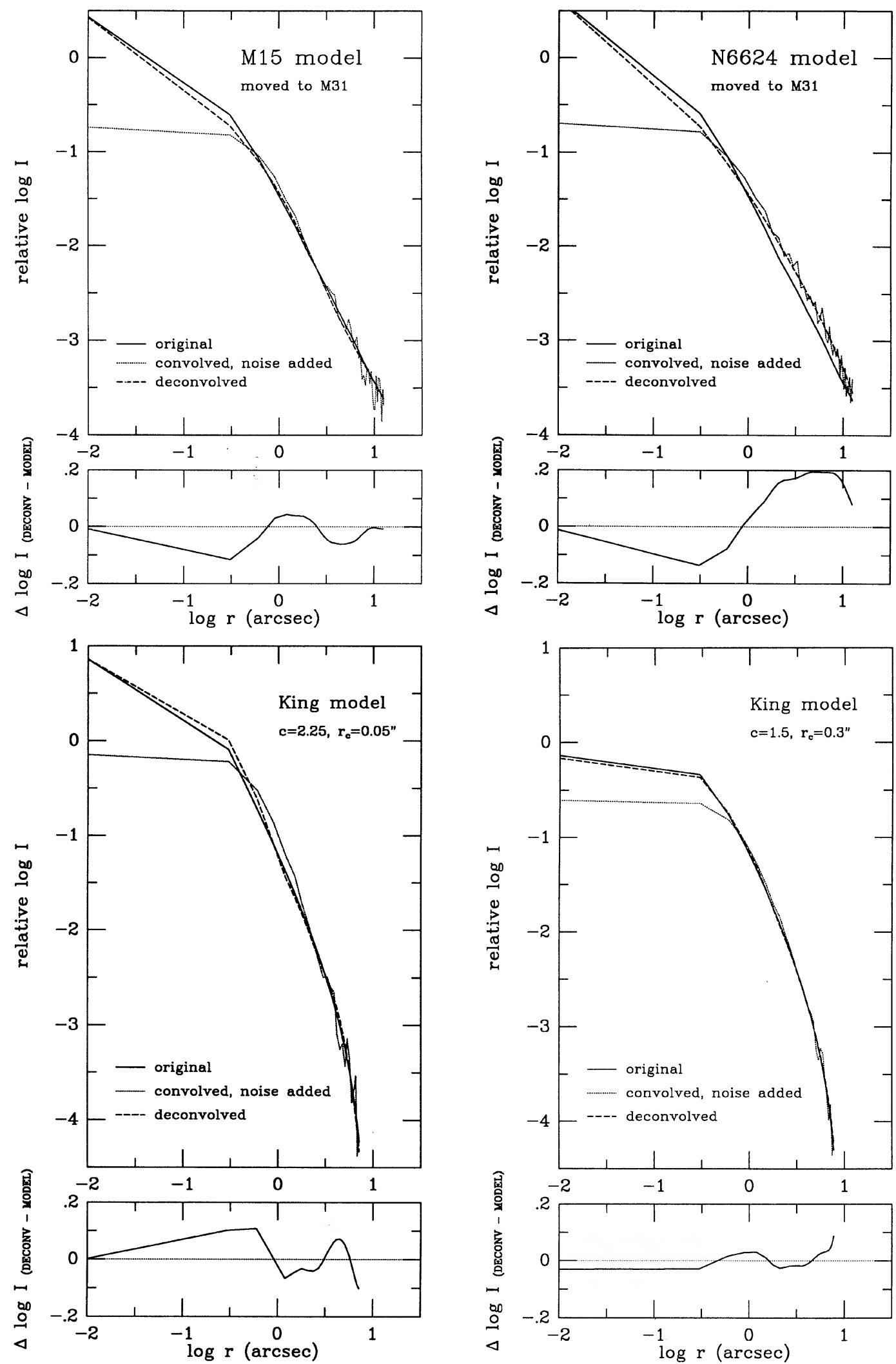

FIG. 2. Feasibility tests, using the models of the two post-core-collapse clusters, "moved" to the distance of M31, and two pure King (1966) models, as described in the text. The original profiles, shown as solid lines, were resampled with the 0.298 arcsec pixels, convolved with the average observed PSF, and degraded with Poissonian noise so as to match the $\mathrm{S} / \mathrm{N}$ of the real data (dotted lines). The results of the deconvolutions, using the RMG method, are shown as dashed lines. Smaller panels show the residuals of the restored profiles from the original ones (note an expanded scale). 


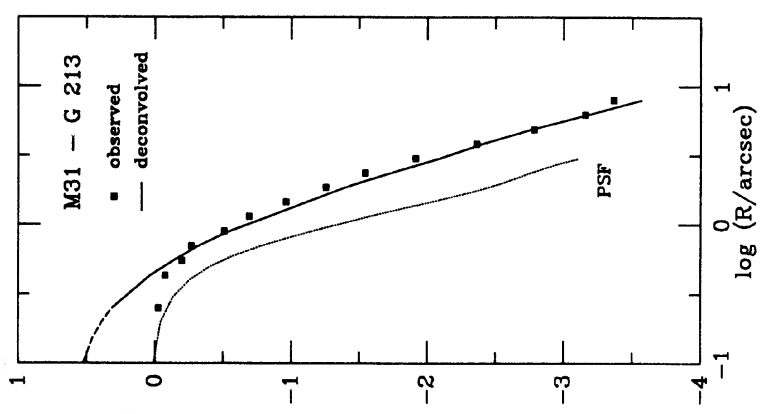

(parsasqo) ${ }^{0} \mathrm{I} / \mathrm{I}$ 8ั0
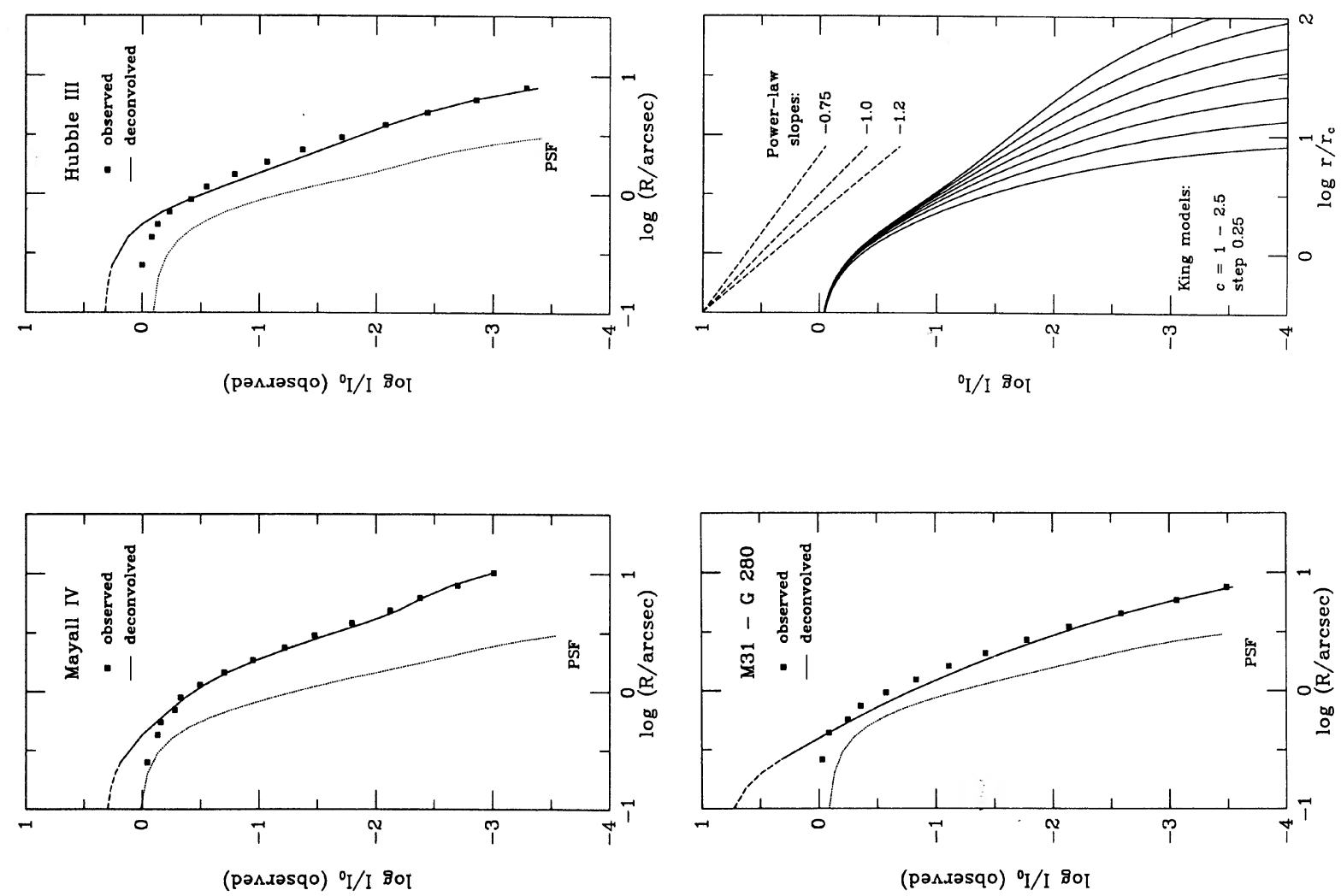


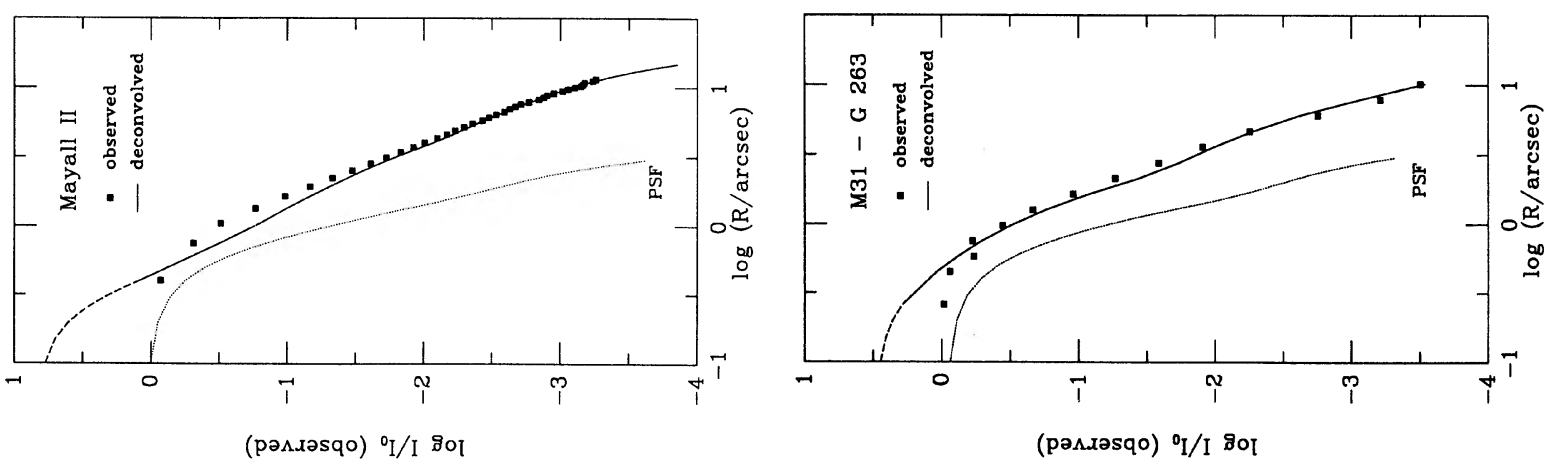
TABLE III. Deconvolved surface-brightness profiles.

\begin{tabular}{|c|c|c|c|c|c|c|}
\hline $\log r(")$ & $\begin{array}{l}\text { Mayall } \\
\text { II }\end{array}$ & $\begin{array}{c}\text { Mayall } \\
\text { IV }\end{array}$ & $\begin{array}{l}\text { Hubble } \\
\text { III }\end{array}$ & G213 & G263 & G280 \\
\hline center & 0.832 & 0.296 & 0.256 & 0.528 & 0.388 & 0.704 \\
\hline-1.0 & 0.771 & 0.273 & 0.244 & 0.465 & 0.356 & 0.613 \\
\hline-0.9 & 0.735 & 0.260 & 0.238 & 0.433 & 0.338 & 0.562 \\
\hline-0.8 & 0.681 & 0.240 & 0.227 & 0.389 & 0.310 & 0.486 \\
\hline-0.7 & 0.598 & 0.210 & 0.210 & 0.329 & 0.269 & 0.376 \\
\hline-0.6 & 0.473 & 0.164 & 0.184 & 0.252 & 0.207 & 0.228 \\
\hline-0.5 & 0.299 & 0.098 & 0.142 & 0.153 & 0.122 & 0.054 \\
\hline-0.4 & 0.082 & 0.009 & 0.079 & 0.025 & 0.013 & -0.125 \\
\hline-0.3 & 0.142 & -0.097 & -0.018 & -0.127 & -0.114 & -0.307 \\
\hline-0.2 & -0.347 & -0.211 & -0.161 & -0.295 & -0.257 & -0.503 \\
\hline-0.1 & -0.555 & -0.329 & -0.358 & -0.492 & -0.423 & -0.704 \\
\hline 0.0 & -0.761 & -0.464 & -0.603 & -0.739 & -0.620 & -0.917 \\
\hline 0.1 & -0.946 & -0.629 & -0.872 & -1.015 & -0.845 & -1.150 \\
\hline 0.2 & -1.135 & -0.836 & -1.145 & -1.274 & -1.117 & -1.389 \\
\hline 0.3 & -1.339 & -1.087 & -1.416 & -1.551 & -1.435 & -1.642 \\
\hline 0.4 & -1.554 & -1.366 & -1.683 & -1.885 & -1.721 & -1.925 \\
\hline 0.5 & -1.802 & -1.663 & -1.948 & -2.217 & -1.951 & -2.216 \\
\hline 0.6 & -2.067 & -1.978 & -2.225 & -2.515 & -2.187 & -2.533 \\
\hline 0.7 & -2.303 & -2.235 & -2.539 & -2.864 & -2.448 & -2.906 \\
\hline 0.8 & -2.534 & -2.434 & -2.928 & -3.261 & -2.769 & -3.303 \\
\hline 0.9 & -2.794 & -2.669 & -3.452 & -3.628 & -3.170 & -3.773 \\
\hline 1.0 & -3.089 & -3.004 & & & -3.592 & \\
\hline 1.1 & -3.480 & & & & & \\
\hline 1.2 & -4.081 & & & & & \\
\hline
\end{tabular}

None of the profiles is well fitted by the single-mass, isotropic King models. Perhaps this is not surprising, given that the clusters are still under-resolved. In addition, the simple King models we use here may be inadequate in describing the real clusters, and anisotropy or other dynamical effects may be important. However, in order to provide at least some approximate estimates of the structural parameters, we fitted the model curves to the deconvolved profiles by sliding them horizontally and vertically on the log-log plots. We allowed for the possibility that the profiles were still unresolved, and thus have larger cores and lower central surface brightness than the models, but match at least approximately the envelope shape. Given the nature of possible systematics and limits of the deconvolution technique and the data, this "eye + brain" technique is probably sufficient, and a more sophisticated, objective(?) approach would hardly be justified. The following estimates should therefore be taken only as a rough guide:

Mayall II: This cluster is clearly unresolved with our data. No good fit, but a possible match with $c \geqslant 2$, and $r_{\mathrm{c}} \leqslant 0.1$ arcsec.

Mayall IV: Possible fit with $c \sim 1.5-2$, and $r_{\mathrm{c}} \sim 0.3$ arcsec.

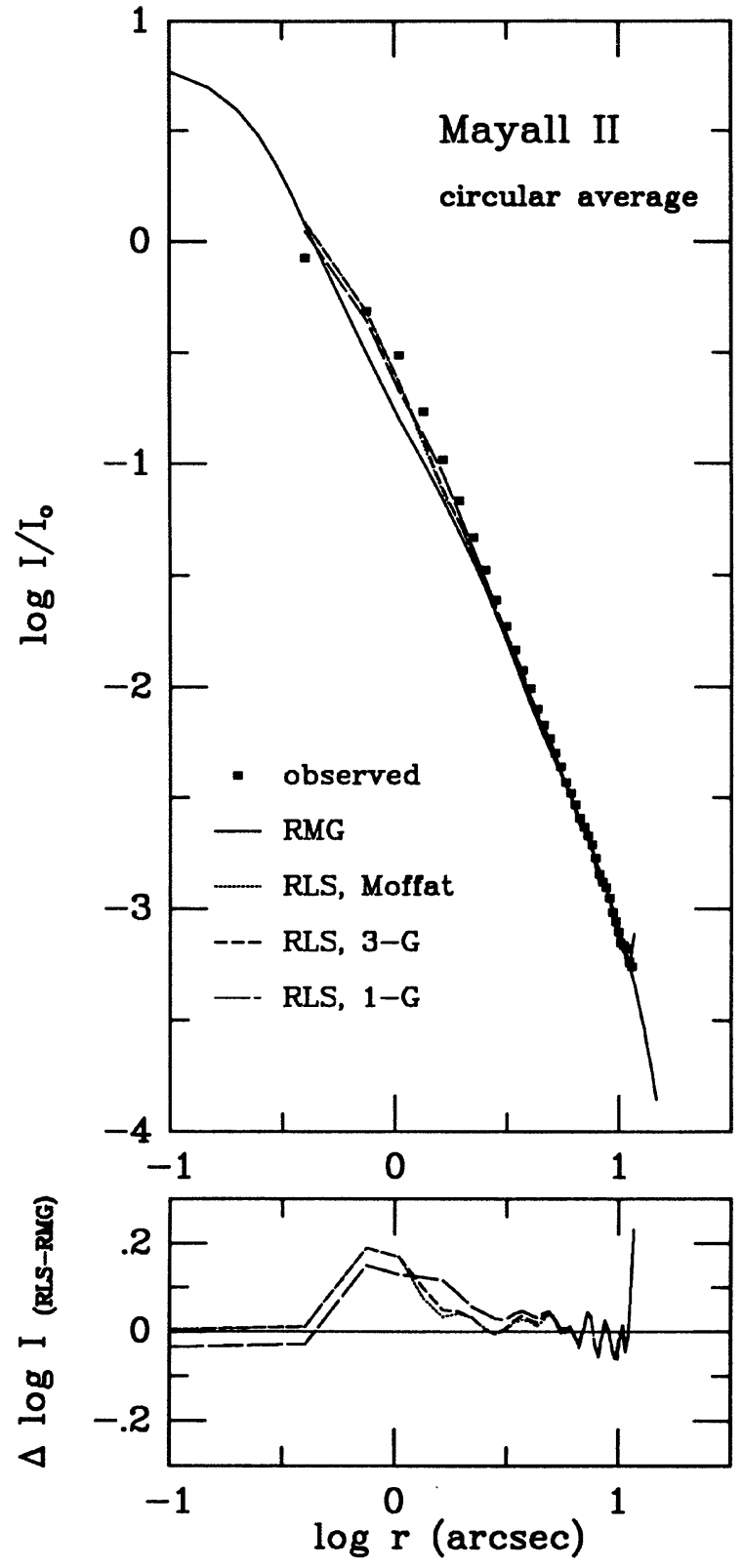

Fig. 4. Comparisons of the deconvolutions using different methods and different PSF representations, as described in the text. The lower panel shows the residuals of the three RLS deconvolutions from the fiducial RMG deconvolution (not an expanded scale).

TABLE IV. Parameters of the deconvolved profiles

\begin{tabular}{|c|c|c|c|c|c|}
\hline & $\mathrm{HWHM}_{\mathrm{obs}}$ & $\mathrm{HWHM}_{\text {decon }}$ & $s_{1}$ & $\Delta \mu_{\text {decon-obs }}$ & Residuals rms \\
\hline $\begin{array}{l}\text { Mayall II } \\
\text { Mayall IV } \\
\text { Hubble III } \\
\text { M31-G213 } \\
\text { M31-G263 } \\
\text { M31-G280 }\end{array}$ & $\begin{array}{l}0.74 \\
0.92 \\
0.78 \\
0.76 \\
0.86 \\
0.70\end{array}$ & $\begin{array}{l}0.24 \\
0.54 \\
0.60 \\
0.45 \\
0.50 \\
0.41\end{array}$ & $\begin{array}{l}0.18 \\
0.23 \\
0.38 \\
0.10 \\
0.18 \\
0.13\end{array}$ & $\begin{array}{l}2.15 \\
0.74 \\
1.64 \\
1.32 \\
0.97 \\
1.76\end{array}$ & $\begin{array}{l}0.012 \\
0.040 \\
0.035 \\
0.036 \\
0.036 \\
0.017\end{array}$ \\
\hline
\end{tabular}






FIG. 5. Comparisons of the deconvolutions using the RLS and the RMG methods, and the surface-brightness profiles derived as the circular and the elliptical isophote averages (note that the sampling is different). The details are given in the text.

Hubble III: Possible fit with $c \sim 1.5-2$, and $r_{\mathrm{c}} \sim 0.2-0.3$ arcsec.

G213: Possible fit with $c \sim 1.5$, and $r_{\mathrm{c}} \sim 0.2$ arcsec.

G263: Possible fit with $c \sim 2$, and $r_{\mathrm{c}} \sim 0.1$ arcsec.

G280: Possible fit with $c \sim 1.5-2$, and $r_{\mathrm{c}} \sim 0.1-0.2$ arcsec.

All core radii estimates should be taken as the upper limits. Still, they are about a factor of 2-3 smaller than the previous estimates by Crampton et al. (1985) or Battistini et al. (1987), which illustrates both the importance of the seeing effects and the improvements that can be achieved using our seeing deconvolution technique.

\section{CONCLUSIONS AND FUTURE PROSPECTS}

The ultimate test of our results will be a comparison of our seeing-deconvolved profiles with future data obtained with the HST. Still, the results obtained here are encouraging: We can study the structure of globular clusters at the distance to M31 at parsec, or even subparsec, resolution. A survey of a couple of hundred globulars in the M31 system is within the reach of ground-based observations. Seeing-deconvolved profiles of such a sample and comparisons with the Galactic system could provide valuable insights into the dynamical evolution of the M31 globular cluster system, and possibly also the mass distribution in this galaxy.

It is interesting to note that in terms of the angular resolution (effective seeing) and sampling, observing the M31 system from the ground is roughly equivalent to observing galaxies in the Virgo Cluster with the HST. This opens interesting possibilities in comparing the globular cluster systems in galaxies of different Hubble types, including M87. For example, the discrepancy in relative specific frequencies of globulars in spiral and elliptical galaxies was often used as an argument against formation of ellipticals by mergers of spirals only; it would be important to know if there are other systematic differences, e.g., in morphological properties of globular clusters in galaxies of different types. In the case of M87, and possibly other giant ellipticals as well, there is an additional puzzle: The core radius of the globular cluster system is much larger than that of the underlying galactic light (Lauer and Kormendy 1986). Ostriker et al. (1989) intepreted this as a sign of dynamical attrition, due to tidal shocks in a triaxial gravitational potential, in analogy with, but perhaps more extreme then, the situation in our galaxy (Chernoff, Kochanek, and Shapiro 1986; Chernoff and Shapiro 1987; Chernoff and Djorgovski 1989). If this explanation is correct, we would expect that the clusters closer to the center of M87 should have postcollapse cores more often, or at least have systematically higher concentrations than the clusters found at larger radii.

Core structure of early-type galaxies is another example of a scientific application of this type. The structure of core surface-brightness profiles often shows deviations from the simple Hubble, de Vaucouleurs, or even the isothermal King model profiles (Light et al. 1974; Kent 1983; Nieto and Vidal 1984; Kormendy 1985; Lauer 1985a,b, etc.). Such deviations reflect dynamical anisotropies of the underlying stellar population. Some cores may also contain dynamically distinct subsystems (Efstathiou et al. 1982; Kormendy 1984; Bender 1988; Franx and Illingworth 1988; Jedrzejewski and Schechter 1988), and some may even harbor supermassive black holes (Tonry 1984; Kormendy 1988a,b, etc.). The systematics of core properties with other global parameters may help us understand formative and evolutionary processes of early-type galaxies and bulges (Lauer 1985b). These issues are reviewed, e.g., by Kormendy and Djorgovski (1989) and references therein. Schweizer $(1979,1981)$ and Djorgovski (1983) attempted to model the seeing effects by assuming the form of the unsmeared profiles. A more ambitious approach was undertaken by Lauer (1985a,b), who used modified Wiener filtering to partly deconvolve the seeing. Techniques used in this paper were already applied to the cores of nearby galaxies by Bendinelli et al. $(1982,1984 b, 1986$, and references therein ). Further systematic studies along these lines are now in progress.

A combination of high-quality data and reliable image restoration techniques can be a powerful tool for astronomical research, and we have yet to see its full potential. We are not advocating overinterpretations - the point is that one should use the full information present, albeit hidden in the data, and that well-understood numerical methods are available to do so.

We are indebted to many colleagues for stimulating and informative discussions. We also thank Dr. Hyron Spinrad and the staff of Kitt Peak National Observatory for their help in obtaining the data used here. S.D. acknowledges the 
hospitality and the stimulating atmosphere of Dipartimento di Astronomia, Università di Bologna, where this paper was prepared. This work was supported in part by the Ministero della Pubblica Istruzione (O.B., F.Z.), by the Osservatorio Astronomico di Bologna (G.P.), and by the Alfred P. Sloan Foundation and California Institute of Technology (S.D.).

\section{REFERENCES}

Battistini, P., Bònoli, F., Braccesi, A., Federici, L., Fusi Pecci, F., Marano, B., and Börngen, F. (1987). Astron. Astrophys. Suppl. 67, 447.

Bender, R. (1988). Astron. Astrophys. 202, L5.

Bendinelli, O. (1989). Astrophys. J. Suppl. (in press).

Bendinelli, O., Di Iorio, A., Parmeggiani, G., and Zavatti, F. (1985). Astron. Astrophys. 153, 265.

Bendinelli, O., Lorenzutta, S., Parmeggiani, G., and Zavatti, F. (1984a). Astron. Astrophys. 138, 337.

Bendinelli, O., Parmeggiani, G., Piccioni, A., and Zavatti, F. (1987a). Astron. J. 94, 1095.

Bendinelli, O., Parmeggiani, G., and Zavatti, F. (1982). Astrophys. Space Sci. 83, 239.

Bendinelli, O., Parmeggiani, G., and Zavatti, F. (1984b). Astron. Astrophys. 140, 174

Bendinelli, O., Parmeggiani, G., and Zavatti, F. (1986). Astrophys. J. 308, 611.

Bendinelli, O., Parmeggiani, G., and Zavatti, F. (1987b). J. Astrophys. Astron. 8, 343 .

Bendinelli, O., Parmeggiani, G., and Zavatti, F. (1988). J. Astrophys. Astron. 9, 17.

Bendinelli, O., Parmeggiani, G., and Zavatti, F. (1989). In Highlights of Astronomy, Proceedings of the 20th IAU General Assembly edited by D. McNally (Kluwer, Dordrecht), p. 657.

Bracewell, R. N. (1979). Annu. Rev. Astron. Astropys. 17, 113.

Brault, J. W., and White, O. R. (1971). Astron. Astrophys. 13, 169.

Brown, G. S. (1973). Univ. Texas Publ. Astron. No. 11.

Bryan, R. K., and Skilling, J. (1980). Mon. Not. R. Astron. Soc. 191, 69.

Chernoff D., and Djorgovski, S. (1989). Astrophys. J. 339, 904.

Chernoff, D., Kochanek, C., and Shapiro, S. (1986). Astrophys. J. 309, 183.

Chernoff, D., and Shapiro, S. (1987). Astrophys. J. 322, 113.

Crampton, D., Cowley, A. P., Schade, D., and Chayer, P. (1985). Astrophys. J. 228, 494.

Coulman, C. E. (1985). Annu. Rev. Astron. Astrophys. 23, 19.

Djorgovski, S. (1983). J. Astrophys. Astron. 4, 271

Djorgovski, S. (1985). Ph.D. thesis, University of California, Berkeley.

Djorgovski, S. (1988). In Globular Cluster Systems in Galaxies, Proceedings of IAU Symposium No. 126, edited by J. Grindlay and A. G. D. Philip (Kluwer, Dordrecht), p. 333.

Djorgovski, S., and King, I. R. (1984). Astrophys. J. Lett. 277, L49.

Djorgovski, S., and King, I. R. (1986). Astrophys. J. Lett. 305, L61.

Efstathiou, G., Ellis, R. S., and Carter, D. (1982). Mon. Not. R. Astron Soc. 201, 975.

Elson, R., Hut, P., and Inagaki, S. (1987). Annu. Rev. Astron. Astrophys. 25, 565 .

Franx, M., and Illingworth, G. D. (1988). Astrophys. J. Lett. 327, L55.

Fried, D. L. (1966). J. Opt. Soc. Am. 56, 1372.

Frieden, B. R. (1972). J. Opt. Soc. Am. 62, 511.

Fusi Pecci, F. (1988). In Globular Cluster Systems in Galaxies, IAU Sym- posium No. 126, edited by J. Grindlay and A. G. D. Philip (Kluwer, Dordrecht), p. 173.

Goodman, J., and Hut, P., Eds. (1985). Dynamics of Star Clusters, IAU Symposium No. 113 (Reidel, Dordrecht).

Grindlay, J., and Philip, A. G. D., Eds. (1988). Globular Cluster Systems in Galaxies, IAU Symposium No. 126 (Kluwer, Dordrecht).

Groetsch, C. W. (1984). The Theory of Tikhonov Regularization for Fredholm Equations of the First Kind (Pitman, London).

Gull, S. F., and Daniel, G. J. (1978). Nature 272, 686.

Hénon, M. (1961). Ann. Astrophys. 24, 369.

Jedrzejewski, R., and Schechter, P. L. (1988). Astrophys. J. Lett. 330, L87.

Kent, S. M. (1983). Astrophys. J. 266, 562.

King, I. R. (1966). Astron. J. 71, 64.

King, I. R. (1971). Publ. Astron. Soc. Pac. 83, 199.

Kormendy, J. (1984). Astrophys. J. 287, 577.

Kormendy, J. (1985). Astrophys. J. Lett. 292, L9.

Kormendy, J. (1988a). Astrophys. J. 325, 128.

Kormendy, J. (1988b). Astrophys. J. 335, 40.

Kormendy, J., and Djorgovski, S. (1989). Annu. Rev. Astron.f Astrophys. 27, 235.

Lauer, T. R. (1985a). Astrophys. J. Suppl. 57, 473.

Lauer, T. R. (1985b). Astrophys. J. 292, 104.

Lauer, T. R., and Kormendy, J. (1986). Astrophys. J. Lett. 303, L1.

Light, E. S., Danielson, R. E., and Schwarzschild, M. (1974). Astrophys. J. 194, 257.

Lugger, P., Cohn, H., Grindlay, J., Bailyn, C., and Hertz, P. (1987). Astrophys. J. 320, 482.

Mateo, M. (1987). Astrophys. J. Lett. 323, L41.

Merritt, D., Ed. (1989). Dynamics of Dense Stellar Systems, Proceedings of CITA Workshop (Cambridge University, Cambridge).

Meylan, G., and Djorgovski, S. (1987). Astrophys. J. Lett. 322, L91.

Moffat, A. F. J. (1969). Astron. Astrophys. 3, 455.

Narayan, R., and Nityananda, R. (1986). Annu. Rev. Astron. Astrophys. 24, 127.

Nieto, J.-L., and Vidal, J.-L. (1984). Mon. Not. R. Astron. Soc. 209, 21 p.

Ostriker, J., Binney, J., and Saha, P. (1989). Mon. Not. R. Astron. Soc. 241, 849.

Sargent, W. L. W., Kowal, S. T., Hartwick, F. D. A., and van den Bergh, S. (1977). Astron. J. 82, 947.

Schweizer, F. (1979). Astrophys. J. 233, 23; 236, 1056 (1980) (E).

Schweizer, F. (1981). Astron. J. 86, 662.

Tonry, J. L. (1984). Astrophys. J. Lett. 283, L27.

de Vaucouleurs, G. (1978). Astrophys. J. 223, 730.

Webbink, R. (1985). In Dynamics of Star Clusters, IAU Symposium No.

113, edited by J. Goodman and P. Hut (Reidel, Dordrecht), p. 541.

Weir, W. N. (1987). Senior thesis, Harvard University.

Wells, D. C. (1980). Proc. Soc. Photo-Opt. Instrum. Eng. 264, 148.

Woolf, N. J. (1982). Annu. Rev. Astron. Astrophys. 20, 367.

Young, A. T. (1974). Astrophys. J. 189, 587. 